\title{
The Effect of Low-Intensity Extracorporeal Shockwave Treatment on the Urinary Bladder in an Experimental Diabetic Rat Model
}

\author{
Fotios Dimitriadis $^{1,2}$, Maria Papaioannou ${ }^{3}$, Ioannis Sokolakis ${ }^{2,4}$, Aikaterini Fragou ${ }^{2}$, Dimitrios Hatzichristou ${ }^{1,2}$, \\ Apostolos Apostolidis ${ }^{2,5}$ \\ ${ }^{1} 1$ st Department of Urology, School of Medicine, Aristotle University of Thessaloniki, Thessaloniki, Greece \\ ${ }^{2}$ Institute for the Study of Urologic Diseases, Aristotle University of Thessaloniki, Thessaloniki, Greece \\ ${ }^{3}$ Laboratory of Biological Chemistry, School of Medicine, Faculty of Health Sciences, Aristotle University of Thessaloniki, Thessaloniki, Greece \\ ${ }^{4}$ Department of Urology, Martha-Maria Hospital Nuremberg, Nuremberg, Germany \\ ${ }^{5}$ 2nd Department of Urology, School of Medicine, Aristotle University of Thessaloniki, Thessaloniki, Greece
}

Purpose: Preclinical data increasingly support an impact of low-intensity extracorporeal shockwave therapy (Li-ESWT) on the bladder. We investigated the molecular effects of Li-ESWT on the bladder of a streptozotocin-induced diabetic rat model. Methods: Fifteen 8-week-old male Wistar rats were randomized into 3 groups: a control group $(n=5)$, a group of diabetic rats without treatment (diabetes mellitus $[\mathrm{DM}], \mathrm{n}=5$ ) and a group of diabetic rats treated with Li-ESWT $(\mathrm{DM}-\mathrm{ESWT}, \mathrm{n}=5)$. A single intraperitoneal dose of streptozotocin $(60 \mathrm{mg} / \mathrm{kg})$ was used to induce diabetes. Twenty days after diabetes induction, each rat in the DM-ESWT group received 300 shockwaves with an energy flux density of $0.09 \mathrm{~mJ} / \mathrm{mm}^{2}$. Sessions were repeated 3 times/week for 2 weeks, followed by a 2-week washout period. Total RNA from bladder tissue was extracted, cDNA was synthesized, and quantitative real-time polymerase chain reaction was performed to analyze the expression pattern of transient receptor potential vanilloid 1 (Trpv1), interleukin-1 $\beta$ (Illb), and the muscarinic receptors M1, M2, and M3 (Chrm1, Chrm2, and Chrm3).

Results: The expression of Trpv1, Illb, and Chrm 2 genes was significantly different between the 3 groups $(\mathrm{P}=0.002, \mathrm{P}<0.0001$, and $\mathrm{P}=0.011$, respectively; 1-way analysis of variance). In the DM group, the expression of all genes was higher than in the control group, but statistical significance was observed only for $\operatorname{Trpv1}$ and $\operatorname{Il} 1 b(\mathrm{P}=0.002$ and $\mathrm{P}<0.0001$, respectively). Li-ESWT significantly reduced the expression of $I l 1 b$ and Chrm $2(\mathrm{P}=0.001$ and $\mathrm{P}=0.011$, respectively), whereas a nonsignificant tendency for reduced expression was noted for Trpv1 $(\mathrm{P}=0.069)$.

Conclusions: The induction of diabetes was associated with increased expression of genes related to mechanosensation, inflammation/ischemia, and contraction in the rat bladder. Li-ESWT reduced the expression of IL1b, Chrm2, and to a lesser extent Trpv1 toward the control levels, suggesting the therapeutic potential of this treatment modality for diabetic cystopathy.

Keywords: Extracorporeal shockwave therapy; Urinary bladder; Diabetes mellitus

- Research Ethics: The experiments were performed under a project license (license number 453673/3391) granted by the Veterinary Directorate of Thessaloniki, Greece, in compliance with the European Directive 2010/63/EEC, the guidelines of the Greek authorities and the Aristotle University of Thessaloniki Ethics committee for the care and use of animals. The animal study was conducted at the Experimental Research Centre of Papageorgiou General Hospital, Thessaloniki, Greece. The molecular studies were conducted in the Laboratory of Biological Chemistry, School of Medicine, Faculty of Health Sciences, Aristotle University of Thessaloniki, Greece.

- Conflict of Interest: Medispec Ltd., Israel provided the device and the applicators for the study. Except for that, no potential conflict of interest relevant to this article was reported.

Corresponding author: Apostolos Apostolidis (iD https://orcid.org/0000-0003-4694-0578 2nd Department of Urology, Aristotle University of Thessaloniki, 'Papageorgiou' General Hospital, Ring Road, Nea Efkarpia 56403 Thessaloniki, Greece E-mail: zefxis@yahoo.co.uk

Submitted: September 17, 2020 / Accepted after revision: November 24, 2020
This is an Open Access article distributed under the terms of the Creative Commons Attribution Non-Commercial License (https://creativecommons.org/licenses/by-nc/4.0/) which permits unrestricted non-commercial use, distribution, and reproduction in any medium, provided the original work is properly cited. 


\begin{abstract}
- HIGHLIGHTS
- Induction of diabetes in a rat model resulted in increased expression of genes related to mechanosensation, inflammation/ischemia, and contraction in the bladder. Li-ESWT reduced the expression of IL 1b, Chrm2, and Trpv1 genes towards controls levels.
\end{abstract}

\section{INTRODUCTION}

Extracorporeal shockwave therapy is a noninvasive treatment method that uses the passage of acoustic waves through the tissue to induce the desired effects. It was originally introduced as a non-invasive treatment for kidney stones and has since been used in the management of many other conditions, including bone fractures, musculoskeletal disorders, wound healing, Peyronie's disease, ischemic cardiovascular disorders, and erectile dysfunction [1,2].

Increasing preclinical evidence supports the role of low-intensity extracorporeal shockwave therapy (Li-ESWT) in the treatment of noninfectious cystitis and detrusor underactivity (DU). In a variety of animal models including cyclophosphamide-induced cystitis, radiation cystitis, uroplakin-induced autoimmune interstitial cystitis, cryoinjury-induced DU, and diabetic underactive bladder, treatment with Li-ESWT was found to improve bladder dysfunction and pain, reduce the inflammatory response, and ameliorate muscle contractility and bladder morphology [3-8].

Diabetic cystopathy or diabetic bladder dysfunction (DBD) is thought to be a result of a 2 -step process. Initially, it presents with compensated bladder hypertrophy and increased contractility due to polyuria. The second stage is theorized to result from an accumulation of toxic metabolites and involves deterioration in voiding function, detrusor muscle decompensation, and ultimately atonic bladder in end-stage patients [9]. The pathophysiology of diabetic cystopathy involves a complex interaction of cellular changes that occur due to hyperglycemia, which can induce cellular changes within the body through oxidative stress and other mechanisms [10]. Excess glucose oxidation through the tricarboxylic acid cycle results in the production of electrons, which contribute to the formation of reactive oxygen species. This may in part explain the pathophysiology of diabetic cystopathy, specifically through the ability of reactive oxygen species to damage neurons, alter urothelial function, and change smooth muscle architecture [10].

Only 2 studies to date have been published on the use of LiESWT in diabetic cystopathy using animal models; in a rat model of diabetic underactive bladder, Li-ESWT enhanced muscle contractile activity of the bladder and urethra, and sig- nificantly restored bladder morphology via an increase of smooth muscle actin expression in the bladder wall and recovery of neuronal integrity and innervation [11]. In another rat model of DBD, Li-ESWT ameliorated voiding function by recruiting endogenous stem cells, resulting in augmented release of nerve growth factor (NGF) and vascular endothelial growth factor (VEGF) [6].

No experimental study has yet investigated the effects of LiESWT on the signaling pathways involved in DBD at the receptor level. We therefore conducted a pilot study to explore the effect of diabetes mellitus (DM) on the expression of receptor genes related to mechanosensation, inflammation/ischemia, and bladder contraction in the rat bladder, as well as the effect of Li-ESWT treatment on the changes induced by diabetes in the rat bladder. Due to the preliminary nature of the study, no functional experiments were performed.

\section{MATERIALS AND METHODS}

\section{Animals}

All animal experiments were performed under a project license (license number: 453673/3391) granted by the Veterinary Directorate of Thessaloniki, Greece, in compliance with the European Directive 2010/63/EEC, the guidelines of the Greek authorities, and the Aristotle University of Thessaloniki Ethics Committee for the care and use of animals. Fifteen adult male Wistar albino rats $(\mathrm{n}=15)$ were housed with a 12:12-hour light/ dark cycle, at $22^{\circ} \mathrm{C} \pm 1{ }^{\circ} \mathrm{C}$ with $60 \%$ humidity, pellet food, and water ad libitum. The rats were randomized into 3 groups. A control group (control bladder [CB]; $n=5$ ), a group of diabetic rats without treatment $(\mathrm{DM}, \mathrm{n}=5)$, and a group of diabetic rats treated with Li-ESWT (DM-ESWT, $n=5$ ). Due to the paucity of similar studies at the time of the protocol's design and submission to the ethical committee, and respecting the principles of the 3 R's (replacement, reduction, and refinement) for animal studies, the sample-size of each study group was set, according to our veterinary authorities and the ethics committee, to the minimum possible number $(n=5)$ to have enough power to produce statistically significant results. For the induction of $\mathrm{DM}$, a single intraperitoneal dose of streptozotocin $(60 \mathrm{mg} / \mathrm{kg})$ was used. A single-dose intraperitoneal injection of $60 \mathrm{mg} / \mathrm{kg}$ 
of streptozotocin in male adult Wistar rats causes extensive necrosis in Langerhans $\beta$-cell islets and in less than 72 hours can induce experimental DM [12]. The intraperitoneal injection was done in a non per oral state. Streptozotocin was dissolved in $10 \mathrm{mM}$ sodium citrate buffer before giving an intraperitoneal injection. The successful induction of diabetes was confirmed by pathologically increased urine glucose levels. The absence of glucose levels in the urine of healthy rats was also checked. The streptozotocin-induced diabetic rat model is the most frequently used model in experimental studies regarding DBD $[10,13]$. In the diabetic groups, marked polyuria was also observed, with a 2-fold increase in daily urine output. Twenty days after the induction of diabetes, each rat in the DM-ESWT group received Li-ESWT treatment. In diabetic cystopathy, both detrusor overactivity (DO) and DU can occur, mostly depending on the duration of diabetes. Despite the lack of functional tests, our diabetic rat model could be more reflective of the initial phase of diabetic cystopathy, which includes bladder hypertrophy and DU $[13,14]$.

\section{Shockwave Treatment}

Twenty days after the induction of diabetes, each rat in the DMESWT group received the shockwave treatment, after they were anesthetized with an intramuscular injection of a mixture of ketamine $(70 \mathrm{mg} / \mathrm{kg}$ of body weight $)$ and xylazine $(6 \mathrm{mg} / \mathrm{kg}$ of body weight). After placement of each animal in a supine position, its lower abdomen was shaved. For the shockwave treatment, an ultrasound gel was applied on the skin and a specially designed shock wave probe for small animals was used (Omnispec ED1000 standard system, Medispec Ltd., Yehud, Israel) [15]. The shockwave probe was placed in contact with the skin on the dorsal surface of the bladder. A total of 300 shockwave pulses at an energy flux density of $0.09 \mathrm{~mJ} / \mathrm{mm}^{2}$ at $2 \mathrm{~Hz}$ were delivered (Fig. 1). This procedure was repeated 3 times per week for 2 weeks (a total of 1,800 shockwave pulses), followed by a 2-week washout period, as was previously described [16]. As no bladder Li-ESWT studies were available when this protocol was designed, we followed a 2-week waiting period before examining the tissue based on the protocols used earlier in rat studies on erectile tissue $[1,17,18]$. Since exposure to ketamine might potentially influence the regulation of both transient potential receptors and inflammatory markers [19], the CB and $\mathrm{DM}$ rats were also anaesthetized with an injection of a mixture of ketamine and xylazine, on the same dates as the DM-ESWT group, and allowed to recover.

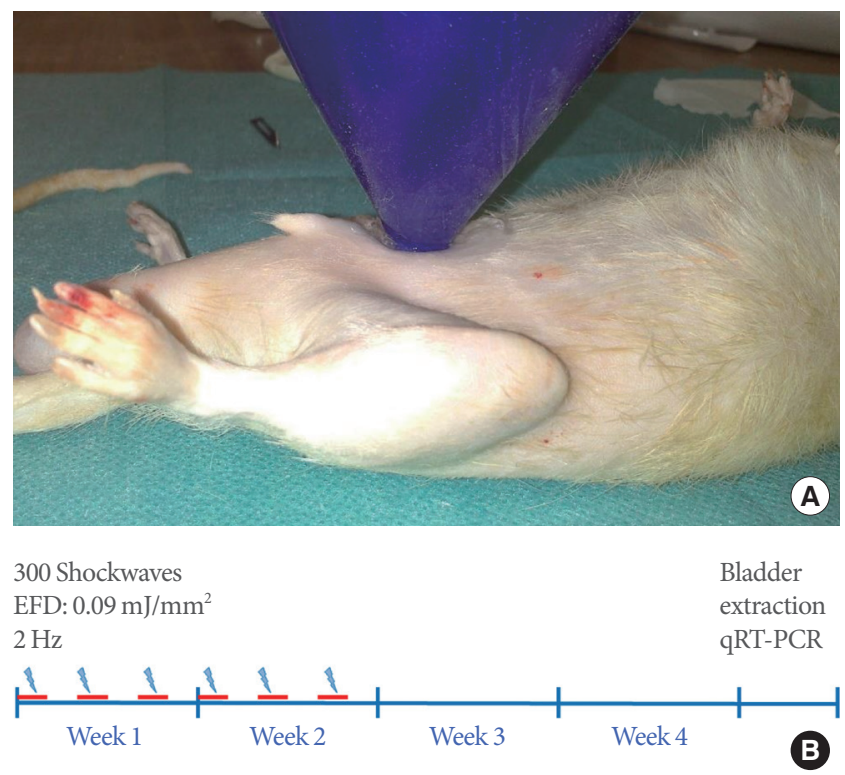

Fig. 1. (A) The application of Li-ESWT in the treatment group on the urinary bladder of an experimental rat. (B) The treatment schedule. Li-ESWT, low-intensity shockwave therapy; EFD, energy flux density; qRT-PCR, quantitative real-time polymerase chain reaction.

\section{RNA Isolation and Real-Time qRT-PCR}

After the experimental period was carried out, the rats were sacrificed and their bladder was harvested. The tissue samples were snap-frozen in liquid nitrogen before their storage at $-80^{\circ} \mathrm{C}$.

The molecular pathways through which Li-ESWT treatment could affect the bladder remain unknown. Therefore, we chose to conduct a preliminary examination of some basic genes regarding bladder function. We examined the muscarinic receptors M1-M3, which play a key role in bladder contraction; the vanilloid receptor, which plays a key role in bladder mechanosensation; and interleukin- $1 \beta$, which is involved in conditions of bladder inflammation and ischemia. For the RNA isolation, the tissue specimens were homogenized to a fine powder using a mortar and pestle in liquid nitrogen and kept at $-80^{\circ} \mathrm{C}$. Total RNA was extracted from tissue with a NucleoSpin RNA Kit (Macherey-Nagel, Bethlehem, PA, USA) according to the manufacturer's instructions. The concentration of the isolated RNA was determined by spectrophotometry. cDNA was synthesized using oligodT and the PrimeScript first strand cDNA synthesis kit (Takara Bio, Shiga, Japan). Quantitative real-time polymerase chain reaction (qRT-PCR) was performed using the Applied Biosystems StepOnePlus real-time PCR system in order to analyze the expression pattern of transient receptor potential vanilloid 
1 (Trpv1) (Rn00583117_m1); interleukin-13 (Illb) (Rn00580432_ $\mathrm{m1}$ ); the muscarinic receptors M1, M2, and M3 (Chrm1, Chrm2, and Chrm3) (Rn00589936_s1, Rn02532311_s1, and Rn00560986_ s1); and the endogenous housekeeping gene Gapdh (Rn01775763_ g1).

In order to compare the amplification products, $\mathrm{Ct}$ data corresponding to the target genes were normalized relative to those of the internal housekeeping gene, Gapdh, and $\Delta \Delta \mathrm{Ct}$ was calculated. Each data point was repeated at least twice. Data were analyzed using IBM SPSS Statistics ver. 25.0 (IBM Co., Armonk, NY, USA) and expressed as mean \pm standard deviation from the mean. Multiple groups were compared using 1-way analysis of variance. Post hoc analysis for comparisons between groups was done using the least significant differences test. Statistical significance was set at $\mathrm{P}<0.05$.

\section{RESULTS}

The expression of the Trpv1, Illb, and Chrm 2 genes was significantly different between the 3 groups $(\mathrm{P}=0.002, \mathrm{P}=0.0001$, and
$\mathrm{P}=0.011$, respectively). The expression of all genes was higher in the DM group than in the $\mathrm{CB}$ group, but the difference was statistically significant only for the Trpv1 and $I l 1 b$ genes $(\mathrm{P}=0.002$ and $\mathrm{P}=0.0001$, respectively). Treatment with Li-ESWT significantly reduced the expression of $I l 1 b$ and Chrm $2(\mathrm{P}=0.001$ and $\mathrm{P}=0.011$, respectively), whereas a non-significant tendency for reduced expression was noted for Trpv1 ( $\mathrm{P}=0.069)$ (Fig. 2A, B, D).

However, the expression of Chrm 1 and Chrm 3 was not significantly different between the 3 groups of animals $(\mathrm{P}=0.084$ and $\mathrm{P}=0.109$, respectively). In the $\mathrm{DM}$ group, there was a weak, but not significant, increase in Chrm 1 and Chrm 3 expression $(\mathrm{P}=$ 0.172 and $\mathrm{P}=0.141$, respectively) compared to the $\mathrm{CB}$ group. Finally, Li-ESWT treatment induced no significant decrease in Chrm 1 and Chrm 3 expression $(\mathrm{P}=0.149$ and $\mathrm{P}=0.344$, respectively) compared to the DM group (Fig. 2C, E).

\section{DISCUSSION}

In this diabetic rat model, we found upregulation of receptor genes associated with bladder mechanosensation, inflamma-
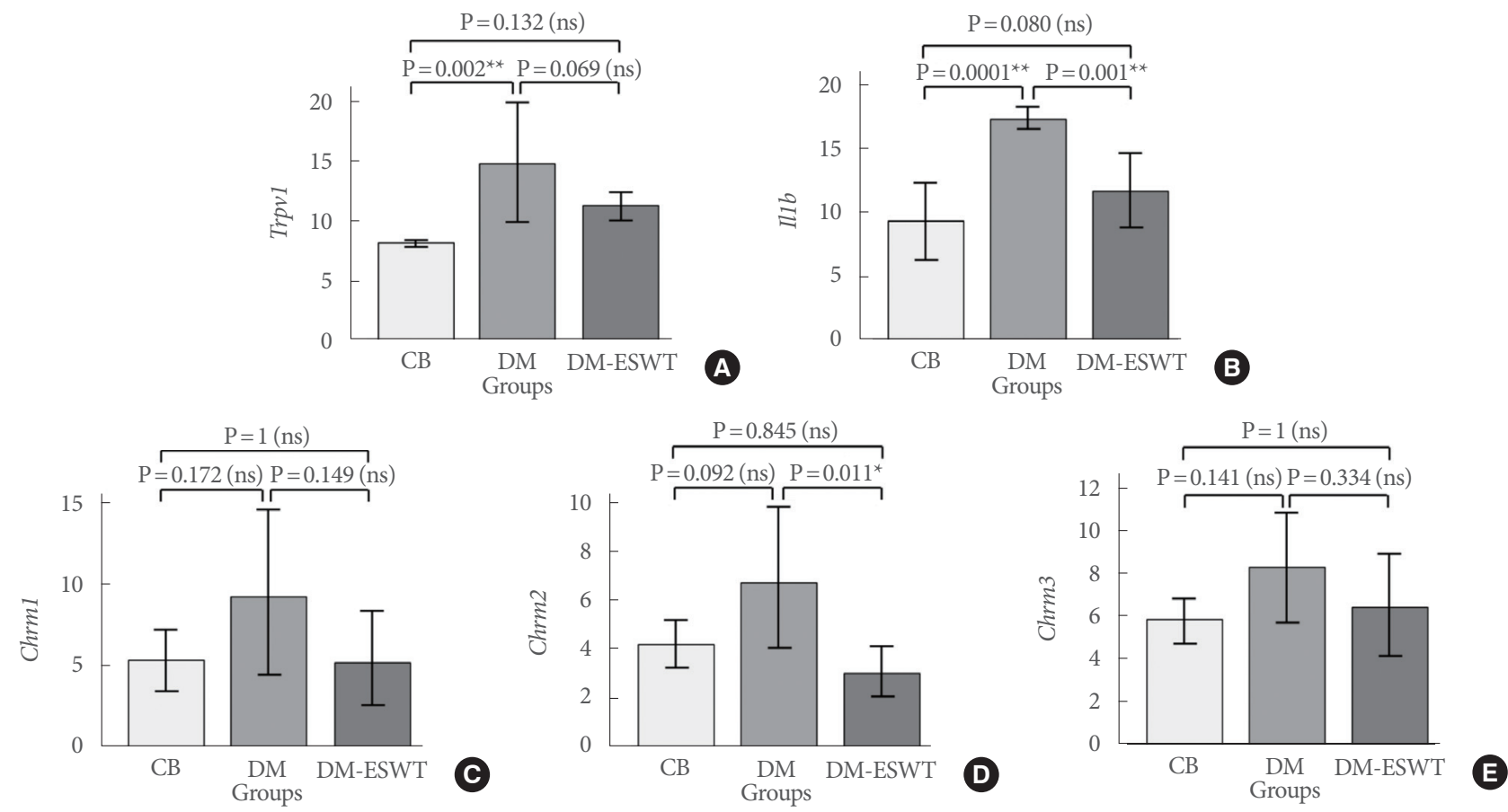

Fig. 2. Histograms of mRNA expression of transient receptor potential vanilloid 1 (Trpv1) (A), interleukin-1 $\beta(\operatorname{Il} 1 b)(B)$, and the muscarinic receptors M1, M2, and M3 (Chrm1, Chrm2, and Chrm3) (C-E) respectively, relative to Gapdh (reference gene), for the 3 experimental animal groups. The $\mathrm{y}$-axis values are the relative quotient as determined by the $\Delta \Delta \mathrm{Ct}$ method from quantitative real-time polymerase chain reaction. Bars show mean values $\pm 95 \%$ confidence intervals. ${ }^{*} \mathrm{P}<0.05$, statistical significance, ${ }^{* *} \mathrm{P}<0.01$, high statistical significance. CB, healthy control group; DM, streptozotocin-induced diabetic control rats; DM-ESWT, streptozotocin-induced diabetic rats treated with low-intensity shockwave therapy; ns, not significant. 
tion/ischemia, and signaling pathways of contraction, adding to previous reports and hypotheses on the pathophysiological mechanisms leading to DBD. Although no functional experiments were carried out in this small pilot study, the upregulation of all receptor genes following the induction of diabetes suggests that the experimental animals entered a phase of bladder overactivity. The vanilloid receptor TRPV1 is involved in the sensation of bladder distention and fullness (mechanosensation) and is abnormally expressed in conditions of DO [20] and bladder pain [21]. Increasing evidence supports an afferently-mediated progression of type $2 \mathrm{DM}$ with sustained lowgrade inflammation via the TRPV1 receptor [22]. In an obese rat model, administration of Trpvl antagonists improved glucose tolerance in parallel with a decrease in inflammatory markers, suggesting that the positive effect of Trpv1 antagonism was exerted via a blockade of low-grade inflammation [23]. In this respect, the $I l 1 b$ receptor gene was significantly upregulated following induction of diabetes in our rat model and significantly downregulated following Li-ESWT treatment. Inflammatory cytokines, such as IL-1 $\beta$, IL-18, and tumor necrosis factor $\alpha$ (TNF- $\alpha$ ) play an important role in both the mediation of the immune response and the upregulation of inflammatory reactions [24]. Although inflammatory processes appear to be involved in the majority of benign urinary pathologies, including diabetes, benign prostatic obstruction, stones, and urinary tract infections, translational research is still scarce [25]. Metabolic-induced inflammation is now thought to be important in both the pathogenesis of diabetes, particularly type $2 \mathrm{DM}$, and its complications [26]. Cytokine levels are increased in patients with diabetes and may contribute to the development of DBD and urinary tract infections. In cultured human bladder smooth muscle cells, the application of a free fatty acid (palmitate) and bacterial lipopolysaccharide was found to upregulate IL- 6 and monocyte chemotactic protein-1 expression and secretion, suggesting a possible link between diabetes and urinary tract infections [27]. Diabetic rats were found to have significantly increased expression of TNF- $\alpha$, IL- $1 \beta$, and IL- 6 in the detrusor smooth muscle tissue. Administration of phosphodiesterase type 4 (roflumilast) was followed by a significant reduction in the expression of those inflammatory cytokines [28]. Another study showed higher levels of IL-18 and IL- $1 \beta$ in the bladder and urethra of diabetic rats than in nondiabetic animals with diverse effects of various antidiabetic agents [29].

Both DO and DU may be found in DM, either separately at different time-points in the progress of the disease, or simulta- neously [30]. In a streptozotocin-induced diabetic rat model, Trpv1 gene expression increased in conjunction with a significant decrease in afferently-mediated bladder contractility [24]. In another streptozotocin-induced diabetic rat model, remodeling of the bladder urothelium occurred with upregulation of the expression of Trpv1, purinergic receptors $\mathrm{P} 2 \mathrm{X} 2 / \mathrm{P} 2 \mathrm{X} 3$, and muscarinic M2 and M3 receptors in the umbrella cells [31]. In an early-stage diabetes rat model, as in our experiment, increased muscarinic and purinergic receptor expression was identified accompanied by enhanced bladder contractile responses, albeit with increased postvoid residual [32]. Bladder ischemia might be a common pathogenetic background for the afferent dysfunction thought to be involved in both DO and DU. In animal models of chronic bladder ischemia, oxidative stress and increased expression of proinflammatory markers in the bladder wall may lead to augmented afferent activity and bladder overactivity [33]. Chronic bladder ischemia has also been proposed to contribute to the pathophysiology of DU via peripheral afferent dysfunction, oxidative stress, and altered muscarinic receptor activity [34].

In our study, Li-ESWT appeared to partly normalize the expression of the examined receptor genes, suggesting a possible mechanism of action for this treatment in DBD. Several preclinical studies have identified molecular changes induced by Li-ESWT in the bladder. In a uroplakin-induced bladder pain model, Li-ESWT significantly decreased the expression and secretion of local and systemic inflammatory markers, including TNF- $\alpha$ and NGF, which increased after uroplakin 3A immunization [7]. In a rat model of cryoinjury-induced DU, Li-ESWT improved the expression of TGF- $\beta 1$, VEGF, and Ki-67 [4]. In a rat model of radiation-induced cystitis, Li-ESWT could exercise a protective role via changes in the bladder protein expression of inflammatory and oxidative stress biomarkers [3]. In CYP-induced cystitis, Li-ESWT suppressed the activation of IL-6, NGF, and COX2 expression, thereby relieving bladder inflammation and overactivity [8]. Finally, in another rat model of DBD, Li-ESWT improved voiding function by recruiting endogenous stem cells, which promoted the release of NGF and VEGF [6].

As mentioned before, both DO and DU may be found in DM, either separately at different time-points in the progress of the disease, or simultaneously [30]. The current treatment options have several limitations. For bladder overactivity, anticholinergics are often the first line treatment. However, side effects and limited effectiveness often lead to treatment discontinua- 
tion [35]. Second-line treatments (e.g., neuromodulation or intravesical injection of botulinum toxin A) are invasive treatments with various efficacy rates and side effects. Pending functional experiments and further mechanistic studies, Li-ESWT could evolve, as in erectile dysfunction, into a novel noninvasive, nonpharmacological treatment that partially restores bladder function in patients with diabetic cystopathy with minimal side effects [1].

It is important to note the limitations of this study. As previously mentioned, the sample size of this study was rather small. This was due to the paucity of relevant studies at the time this study was designed, and to the application of the reduction principle of the 3 R's for animal studies [36], corresponding to the use of the minimum number of animals in a study in order to reach enough power for statistically significant results. Larger studies are needed to validate our results and to further investigate the impact of Li-ESWT on the urinary bladder. The lack of functional tests is another major limitation of this study. Functional tests have already shown an effect of Li-ESWT on the diabetic bladder in 2 previously published studies [6,11]. As the mechanism of action of Li-ESWT in the diabetic bladder is still unclear, we conducted this preliminary study without concomitant functional tests in order to see how Li-ESWT might affect the diabetic bladder at the molecular level of important mechanistic pathways. We plan to conduct further research exploring the mechanism of action in parallel with functional tests. Finally, this animal model reflects changes of early-stage diabetes. Experiments at further time-points might be able to provide more information on both the time-sequence of DBD and the potential therapeutic effect of various Li-ESWT protocols.

In conclusion, in this rat model, streptozotocin-induced early-stage diabetes appears to affect the bladder at a molecular level by increasing the expression of receptors related to mechanosensation, inflammation/ischemia, and signalling pathways of bladder contraction. Treatment of the diabetic rat bladder with Li-ESWT reduced the expression of Illb, Chrm2, and, to a lesser degree, Trpv1 receptor genes towards the control levels, suggesting a therapeutic potential of this treatment modality for DBD. Certainly, more molecular/signaling pathways could be involved in the changes of DM cystopathy (glucose metabolism, cell survival and proliferation, cell-signaling receptors, cell stress, and cell death), and further research could be designed to explore these pathways in addition to those investigated in this pilot study.

\section{AUTHOR ORCID}

$\begin{array}{ll}\text { Fotios Dimitriadis } & 0000-0003-1198-9144 \\ \text { Maria Papaioannou } & 0000-0003-3149-6523 \\ \text { Ioannis Sokolakis } & 0000-0002-1938-8004 \\ \text { Aikaterini Fragou } & 0000-0002-3443-6908 \\ \text { Dimitrios Hatzichristou } & 0000-0001-6705-3965 \\ \text { Apostolos Apostolidis } & 0000-0003-4694-0578\end{array}$

\section{AUTHOR CONTRIBUTION STATEMENT}

\author{
- Conceptualization: FD, IS, DH, AA \\ - Data curation: FD, MP, IS, AF, AA \\ - Formal analysis: MP, IS, AF \\ - Funding acquisition: DH, AA \\ - Methodology: FD, IS, DH, AA \\ - Project administration: FD, DH, AA \\ - Visualization: FD, DH \\ -Writing-original draft: FD, MP, IS, AF, AA \\ -Writing-review \& editing: FD, MP, IS, DH, AA
}

\section{REFERENCES}

1. Sokolakis I, Dimitriadis F, Teo P, Hatzichristodoulou G, Hatzichristou D, Giuliano F. The basic science behind low-intensity extracorporeal shockwave therapy for erectile dysfunction: a systematic scoping review of pre-clinical studies. J Sex Med 2019;16:168-94.

2. Sokolakis I, Hatzichristodoulou G. Clinical studies on low intensity extracorporeal shockwave therapy for erectile dysfunction: a systematic review and meta-analysis of randomised controlled trials. Int J Impot Res 2019;31:177-94.

3. Chen YT, Chen KH, Sung PH, Yang CC, Cheng BC, Chen CH, et al. Extracorporeal shock wave markedly alleviates radiation-induced chronic cystitis in rat. Am J Transl Res 2018;10:1036-52.eCollection 2018.

4. Chuang YC, Tyagi P, Luo HL, Lee WC, Wang HJ, Huang CC, et al. Long-term functional change of cryoinjury-induced detrusor underactivity and effects of extracorporeal shock wave therapy in a rat model. Int Urol Nephrol 2019;51:617-26.

5. Chuang YC, Tyagi P, Wang HJ, Huang CC, Lin CC, Chancellor MB. Urodynamic and molecular characteristics of detrusor underactivity in a rat cryoinjury model and effects of low energy shock wave therapy. Neurourol Urodyn 2018;37:708-15.

6. Jin Y, Xu L, Zhao Y, Wang M, Jin X, Zhang H. Endogenous stem cells were recruited by defocused low-energy shock wave in treat- 
ing diabetic bladder dysfunction. Stem Cell Rev Rep 2017;13:28798.

7. Li H, Zhang Z, Peng J, Xin Z, Li M, Yang B, et al. Treatment with low-energy shock wave alleviates pain in an animal model of uroplakin 3A-induced autoimmune interstitial cystitis/painful bladder syndrome. Investig Clin Urol 2019;60:359-66.

8. Wang HJ, Lee WC, Tyagi P, Huang CC, Chuang YC. Effects of low energy shock wave therapy on inflammatory moleculars, bladder pain, and bladder function in a rat cystitis model. Neurourol Urodyn 2017;36:1440-7.

9. Wittig L, Carlson KV, Andrews JM, Crump RT, Baverstock RJ. Diabetic bladder dysfunction:a review. Urology 2019;123:1-6.

10. Daneshgari F, Liu G, Birder L, Hanna-Mitchell AT, Chacko S. Diabetic bladder dysfunction: current translational knowledge. J Urol 2009;182(6 Suppl):S18-26.

11. Wang HS, Oh BS, Wang B, Ruan Y, Zhou J, Banie L, et al. Low-intensity extracorporeal shockwave therapy ameliorates diabetic underactive bladder in streptozotocin-induced diabetic rats. BJU Int 2018;122:490-500.

12. Yakhchalian N. Hematological and serum biochemical analysis of streptozotocin-induced insulin dependent diabetes mellitus in male adult wistar rats. bioRxiv 2018:359844. https://doi.org/10. $1101 / 359844$.

13. Arioglu Inan E, Ellenbroek JH, Michel MC. A systematic review of urinary bladder hypertrophy in experimental diabetes: Part I. Streptozotocin-induced rat models. Neurourol Urodyn 2018;37: 1212-9.

14. Cao N, Gu B, Gotoh D, Yoshimura N. Time-dependent changes of urethral function in diabetes mellitus: a review. Int Neurourol J 2019;23:91-9.

15. Assaly-Kaddoum R, Giuliano F, Laurin M, Gorny D, Kergoat M, Bernabé J, et al. Low intensity extracorporeal shock wave therapy improves erectile function in a model of type ii diabetes independently of NO/cGMP pathway. J Urol 2016;196:950-6.

16. Sokolakis I, Dimitriadis F, Psalla D, Karakiulakis G, Kalyvianakis D, Hatzichristou D. Effects of low-intensity shock wave therapy (LiST) on the erectile tissue of naturally aged rats. Int J Impot Res 2019;31: 162-9.

17. Liu T, Shindel AW, Lin G, Lue TF. Cellular signaling pathways modulated by low-intensity extracorporeal shock wave therapy. Int J Impot Res 2019;31:170-6.

18. Qiu X, Lin G, Xin Z, Ferretti L, Zhang H, Lue TF, et al. Effects of low-energy shockwave therapy on the erectile function and tissue of a diabetic rat model. J Sex Med 2013;10:738-46.

19. Lee WC, Tain YL, Chuang YC, Tsai CN, Yu CC, Su CH. Ba-Wei-
Die-Huang-Wan (Hachimi-jio-gan) can ameliorate ketamine-induced cystitis by modulating neuroreceptors, inflammatory mediators, and fibrogenesis in a rat model. Neurourol Urodyn 2019;38: 2159-69.

20. Apostolidis A, Popat R, Yiangou Y, Cockayne D, Ford AP, Davis JB, et al. Decreased sensory receptors P2X3 and TRPV1 in suburothelial nerve fibers following intradetrusor injections of botulinum toxin for human detrusor overactivity. J Urol 2005;174:977-82.

21. Mukerji G, Yiangou Y, Agarwal SK, Anand P. Transient receptor potential vanilloid receptor subtype 1 in painful bladder syndrome and its correlation with pain. J Urol 2006;176:797-801.

22. Gram DX, Holst JJ, Szallasi A. TRPV1: a potential therapeutic target in type 2 diabetes and comorbidities? Trends Mol Med 2017; 23:1002-13.

23. Gram DX, Fribo J, Nagy I, Gotfredsen C, Charrua A, Hansen JB, et al. TRPV1 antagonists as novel anti-diabetic agents: regulation of oral glucose tolerance and insulin secretion through reduction of low-grade inflammation? Med Sci (Basel) 2019;7:82.

24. Sharopov BR, Gulak KL, Philyppov IB, Sotkis AV, Shuba YM. TRPV1 alterations in urinary bladder dysfunction in a rat model of STZ-induced diabetes. Life Sci 2018;193:207-13.

25. Purves JT, Hughes FM Jr. Inflammasomes in the urinary tract: a disease-based review. Am J Physiol Renal Physiol 2016;311:F65362.

26. Wada J, Makino H. Innate immunity in diabetes and diabetic nephropathy. Nat Rev Nephrol 2016;12:13-26.

27. Oberbach A, Schlichting N, Heinrich M, Till H, Stolzenburg JU, Neuhaus J. Free fatty acid palmitate impairs the vitality and function of cultured human bladder smooth muscle cells. PLoS One 2012;7:e41026.

28. Ding H, Zhang P, Li N, Liu Y, Wang P. The phosphodiesterase type 4 inhibitor roflumilast suppresses inflammation to improve diabetic bladder dysfunction rats. Int Urol Nephrol 2019;51:253-60.

29. Shin SJ, Chung S, Kim SJ, Lee EM, Yoo YH, Kim JW, et al. Effect of sodium-glucose co-transporter 2 inhibitor, dapagliflozin, on renal renin-angiotensin system in an animal model of type 2 diabetes. PLoS One 2016;11:e0165703.

30. Lin TL, Chen GD, Chen YC, Huang CN, Ng SC. Aging and recurrent urinary tract infections are associated with bladder dysfunction in type 2 diabetes. Taiwan J Obstet Gynecol 2012;51:381-6.

31. Hanna-Mitchell AT, Ruiz GW, Daneshgari F, Liu G, Apodaca G, Birder LA. Impact of diabetes mellitus on bladder uroepithelial cells. Am J Physiol Regul Integr Comp Physiol 2013;304:R84-93.

32. Yoshizawa T, Hayashi Y, Yoshida A, Yoshida S, Ito Y, Yamaguchi K, et al. Concomitant alteration in number and affinity of P2X and 
muscarinic receptors are associated with bladder dysfunction in early stage of diabetic rats. Int Urol Nephrol 2018;50:451-8.

33. Andersson KE, Nomiya M, Yamaguchi O. Chronic pelvic ischemia: contribution to the pathogenesis of lower urinary tract symptoms (LUTS): a new target for pharmacological treatment? Low Urin Tract Symptoms 2015;7:1-8.

34. Andersson KE, Nomiya M, Sawada N, Yamaguchi O. Pharmacological treatment of chronic pelvic ischemia. Ther Adv Urol 2014;6:
105-14.

35. Chancellor MB, Migliaccio-Walle K, Bramley TJ, Chaudhari SL, Corbell C, Globe D. Long-term patterns of use and treatment failure with anticholinergic agents for overactive bladder. Clin Ther 2013;35:1744-51.

36. Richter V, Muche R, Mayer B.J How much confidence do we need in animal experiments? Statistical assumptions in sample size estimation. Appl Anim Welf Sci 2018;21:325-33. 